\title{
Frequency support among asynchronous AC systems through VSCs emulating power plants
}

\author{
L. Papangelis*, X. Guillaud ${ }^{\dagger}$, T. Van Cutsem ${ }^{\circ}$ \\ *University of Liège, Belgium (1.papangelis@ulg.ac.be) \\ ${ }^{\dagger}$ Ecole Centrale de Lille, France (xavier.guillaud@ec-lille.fr) \\ ${ }^{\circ}$ Fund for Scientific Research (FNRS) and University of Liège, Belgium (t.vancutsem@ulg.ac.be)
}

Keywords: HVDC grid, voltage source converter, frequency support, speed governor, speed droop, integral control

\begin{abstract}
This paper proposes a new control scheme for the Voltage Source Converters (VSCs) of a High Voltage Direct Current (HVDC) grid connecting asynchronous AC systems, so that the latter can mutually support their frequencies after a disturbance. The scheme involves no communication between VSCs. It is activated upon detection of a significant frequency deviation, with the possibility to limit the support requested from other AC systems. It relies on an integral control enforcing the frequency droop characteristics (of classical speed governors), in addition to the voltage-droop control of VSCs. The controller is tuned to emulate the response of a power plant, while avoiding excessive transients in the HVDC system, and limiting the impact on the other AC systems.
\end{abstract}

\section{Introduction}

The increasing need for bulk power transfer over long distances led by the shift to renewable energy production and the gradual replacement of conventional power plants has led to the development of HVDC systems. As is well known, DC is more attractive than $\mathrm{AC}$ transmission for long distances, for submarine connections or for interconnecting two AC systems, when their synchronization would result in excessive inter-area oscillations. Furthermore, an HDVC link between two asynchronous AC systems acts as a "firewall", preventing large disturbances to propagate from one system to the other.

A number of point-to-point links between synchronous or asynchronous areas are already in operation worldwide [1]. The vast majority of the point-to-point connections utilize line commutated converters which, compared with the more recently developed VSCs, have higher capacity and fewer losses. However, VSCs offer more options in terms of controllability, such as four quadrant operation, reversal of power without need to reverse DC voltage polarity, and black start capability. These options make the VSC a very suitable candidate for the development of future HVDC grids.

Once the current challenge of clearing faults in the DC grid will be properly dealt with [2], the aforementioned technolo- gies are expected to lead to AC/DC transmission systems including HVDC grids connecting two or more AC asynchronous systems and/or remote wind farms. The approach proposed in this paper fits that general structure. Of course, it also applies to the traditional HDVC link between two asynchronous areas.

HVDC grids can also provide auxiliary services to AC grids. One of them, and the subject of this paper, is the provision of frequency support. This requires adding a dedicated control to VSCs, which are otherwise immune to changes in the frequency of the AC systems that they connect. The overall approach followed in this paper is to control HVDC system as an "emergency" primary reserve activated in case of significant frequency deviations as also suggested in [3]. For small deviations, the HVDC system remains inactive, to prevent continuous interactions between the frequency controls of AC systems which were otherwise planned to operate asynchronously. In response to larger frequency deviations, the HVDC grid emulates a conventional power plant. To this purpose, it acts as a "hub" to share the primary reserves of the various connected AC systems. However, in accordance with the already mentioned firewall principle, following a power shortage in one of the $\mathrm{AC}$ systems, (i) a limit should be put on the power requested from the other AC systems, and (ii) electromechanical oscillations should not be propagated to these systems.

The simplest and most widespread in the literature implementation consists of adding a frequency droop (proportional) term that enables the VSC to react to frequency deviations by modifying its active power set-point, as proposed in $[4,5,6]$. In Ref. [7], a frequency droop control scheme is proposed to enable offshore wind farms to provide frequency support without communication. A similar implementation is proposed in [8] where the frequency support by the onshore converters is activated replacing the DC voltage droop control when a "disturbed" condition is identified. The so-called "undead-band" (or piecewise) droop scheme proposed in [9] is a variant of the frequency droop control in which the droop varies according to the severity of the under-frequency event. The frequency droop controller and its effect on disturbance propagation have been investigated in [10]. The authors show that the action of the frequency droop control leads to coupling the AC systems frequencies by transferring a fraction of an under-frequency disturbance from one system to the other. 
Frequency droop control enables a fast response by the VSC. However, it has some drawbacks. First, it may not always lead to the same steady-state participation of the VSC due to its interaction with the DC voltage control [5]. Next, by using a simple proportional control, the VSC will follow almost instantaneously the system frequency response, which can lead to significant overshoots during the frequency dip. Finally, by stiffly coupling the asynchronous AC systems, it contributes to disturbance propagation.

The approach proposed in this paper relies on an integral control enforcing the frequency droop characteristics and complementing the classical voltage-droop control of VSCs. This integral control addresses the first issue, since it enforces the desired steady-state participation of the VSC regardless of the DC voltage control characteristics of the system. Moreover, it is shown that by properly tuning the controller gain, the transient response can be "shaped" as desired in the AC systems (e.g. avoiding power injection overshoot) as well as in HVDC grid (e.g. limiting the DC voltage dip).

The rest of the paper is organized as follows. In Section 2, the modelling of the VSC controller and its standard controls are described. The proposed frequency control scheme is presented in Section 3, while the tuning of its parameters is discussed in Section 4. Simulation results obtained with two DCconnected asynchronous AC systems are presented in Section 5. Concluding remarks are offered in Section 6.

\section{Modelling and control of HVDC grid}

This section describes the model used for the DC lines or cables, the VSCs and their standard controls. This model is intended for dynamic simulations in phasor mode (quasisinusoidal approximation [11]). The degree of detail is more than sufficient for studies of frequency dynamics.

\subsection{DC line/cable model}

The branches of the HVDC grid model consist only of resistances. This simplification is justified by the fact that the DC network dynamics are much faster than the dynamics of concern. Thus, the series inductances are neglected, while the shunt capacitances are accounted for in the terminal capacitors, as shown in Fig. 1.

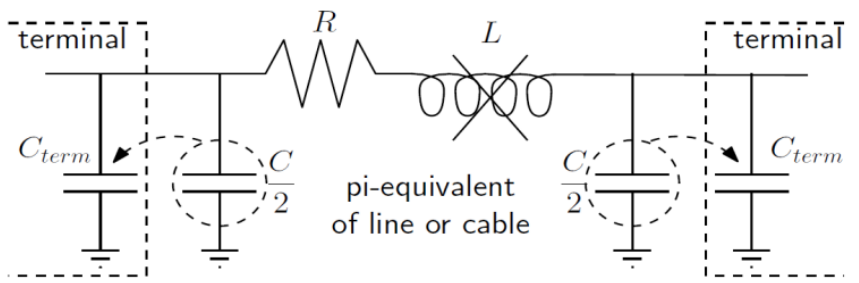

Figure 1: Simplifying the DC line or cable model

\subsection{Converter model}

The overall VSC structure is shown in Fig. 2. Its model is largely inspired of the material presented in Refs. [12,13].

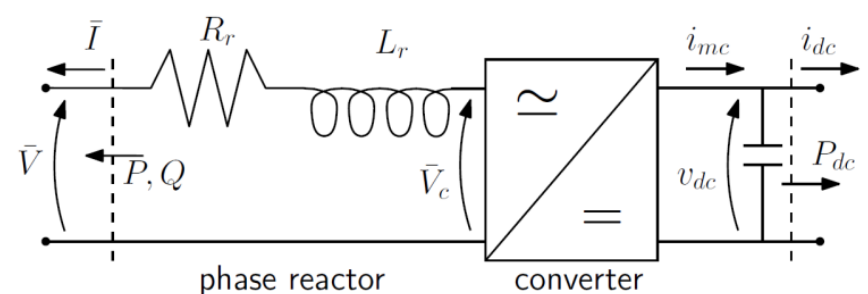

Figure 2: Overall VSC structure

In order to decouple active and reactive power controls, the $d q$ reference frame is used, with the $d$ axis aligned with the AC voltage phasor $\bar{V}$, as shown in Fig. 3. In this figure, $x$ and $y$ denote the orthogonal, rotating axes used to project all phasors. The $d$ and $q$ axes rotate at the angular speed $\omega$. The delay introduced by the phase lock loop, in the order of a few milliseconds, is neglected.

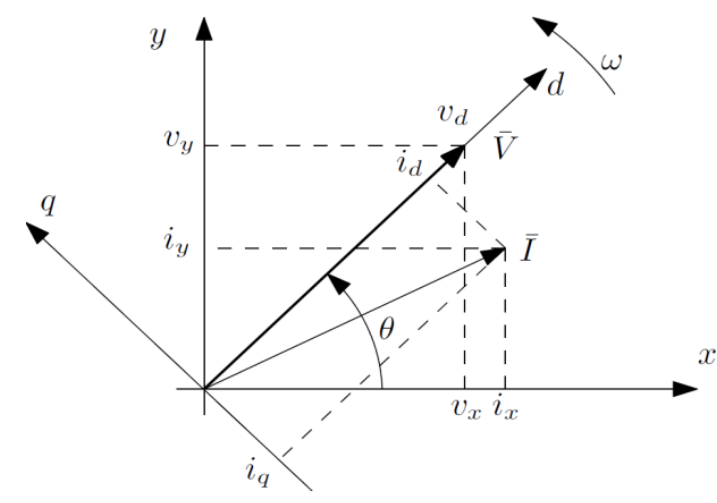

Figure 3: $x y$ and $d q$ reference frames

From Fig. 3 the following equations can be easily derived:

$$
\begin{gathered}
v_{x}=v_{d} \cos \theta \\
v_{y}=v_{d} \sin \theta \\
i_{x}=i_{d} \cos \theta-i_{q} \sin \theta \\
i_{y}=i_{d} \sin \theta+i_{q} \cos \theta
\end{gathered}
$$

where all variables are defined in Fig. 3.

By decomposing the converter voltage phasor $\bar{V}_{c}$ into its $v_{c d}$ and $v_{c q}$ components, the phase reactor equations can be obtained as [13]:

$$
\begin{gathered}
\frac{d i_{d}}{d t}=-\frac{R_{r}}{L_{r}} i_{d}+\omega i_{q}+\frac{1}{L_{r}}\left(v_{c d}-v_{d}\right) \\
\frac{d i_{q}}{d t}=-\frac{R_{r}}{L_{r}} i_{q}+\omega i_{d}+\frac{1}{L_{r}} v_{c q}
\end{gathered}
$$

The dynamics of the DC capacitor are described by:

$$
\frac{d v_{d c}}{d t}=\frac{i_{m c}-i_{d c}}{C_{d c}}
$$

where $v_{d c}$ is the capacitor voltage, the currents $i_{m c}$ and $i_{d c}$ are defined in Fig. 2, and $C_{d c}$ accounts for effects mentioned in Section 2.1.

Finally, neglecting the switching losses, the converter power balance can be written as:

$$
P_{d c}+P=v_{d c} i_{d c}+v_{c d} i_{d}+v_{c q} i_{c q}=0
$$




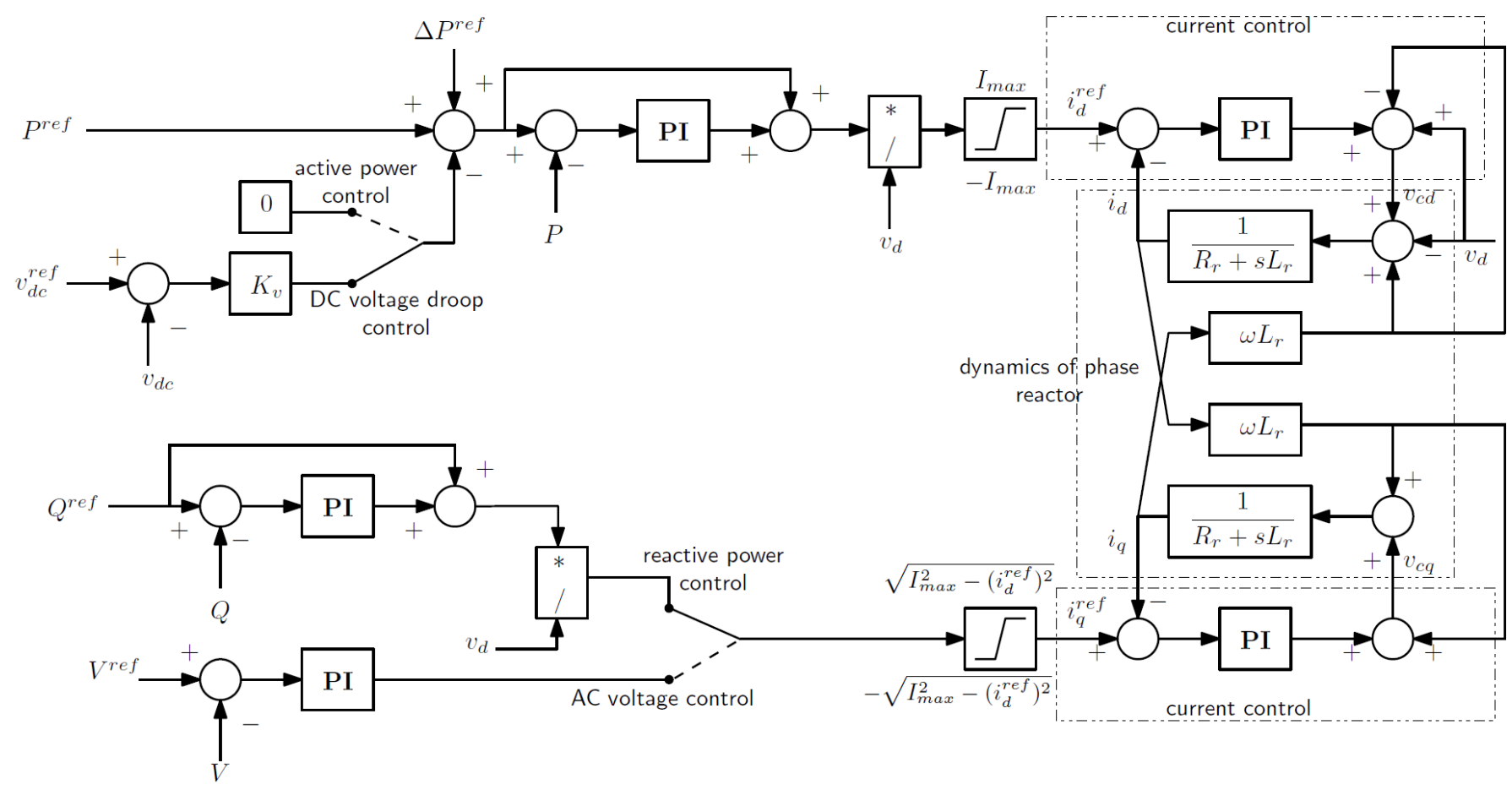

Figure 4: Controls acting on the converter. PI stands for "Proportional-Integral control". $\Delta P^{r e f}$ is the output of the frequency support control detailed in Fig. 5

\subsection{Controls acting on the converter}

The various control loops acting on the VSC are shown in Fig. 4. The active and reactive current components $i_{d}$ and $i_{q}$ are used to control the active and reactive power, respectively. Independent control is achieved by introducing a feedforward term compensating for the coupling induced by the phase reactor and acting on the respective reference values $\left(i_{d}^{r e f}\right.$ and $\left.i_{q}^{r e f}\right)$. The reference value of each current component depends on the control strategy. For $i_{d}^{r e f}$ a choice can be made between active power control and DC voltage droop control. Similarly, for $i_{q}^{r e f}$ a choice can be made between reactive power and AC voltage control.

The current limits account for the thermal capacity of the conductor. In this study it was chosen to give priority to the active current component, resulting in the following limits:

$$
\left|i_{d}^{r e f}\right| \leq I_{\max } \text { and }\left|i_{q}^{r e f}\right| \leq \sqrt{I_{\max }^{2}-\left(i_{d}^{r e f}\right)^{2}}
$$

\subsection{DC voltage control strategies}

Proper operation of an HVDC network requires keeping the voltages at all its nodes in acceptable ranges. This can be achieved by balancing the active power injected in and extracted from the HVDC grid. As shown in [14], the control of $\mathrm{DC}$ voltages is analogous to primary frequency control in AC systems. Specifically, a continuously decreasing DC voltage is evidence of a deficit of active power in the DC grid (i.e. the power injected by the rectifiers is lower than the power extracted by the inverters), in the same way as a deteriorating frequency is evidence of a deficit of active power in an AC system. However, while there is a large kinetic energy stored in the rotating masses of AC power plants, there is comparatively a much smaller electrostatic energy stored in DC capacitors. Therefore, DC voltage control has to be very fast since even a small imbalance of power can lead to discharging the DC capacitors.

Various methods to control the DC voltage of an HVDC grid can be found in the literature (e.g. [14] and its references). This paper focuses on HVDC grids with a combination of VSCs operating either in constant power mode (i.e. no DC voltage control) or in DC voltage-droop mode. The latter is inspired by primary frequency control in an AC system. Each VSC is equipped with a proportional controller and is given an active power and a DC voltage set-point, denoted respectively $P^{r e f}$ and $v_{d c}^{r e f}$. At any operating point its active power $P$ and its DC voltage $v_{d c}$ are linked through:

$$
P=P^{r e f}-K_{v}\left(v_{d c}^{r e f}-v_{d c}\right)
$$

With this control any active power imbalance in the HVDC grid is shared between multiple VSCs.

\section{Principle of the frequency support control}

The proposed frequency controller is shown in Fig. 5. It uses a frequency measurement as input, while its output is a correction $\Delta P^{r e f}$ added to the active power control of the VSC, as shown in Fig. 4 (see upper left part).

The control is inactive as long as frequency remains in a deadband, i.e. it is activated for a large enough frequency 
deviation. Alternatively, it could be triggered by the rate of change of frequency. In Fig. 5, $f$ is the measured frequency and $P$ the active power flow in the VSC. Assuming that activation takes place at $t=t^{*}$, the output of the "memory" block is frozen at the value $P\left(t^{*}\right)$ as suggested by the left switch in the figure. At the same time $t^{*}$, the integral control is activated as suggested by the right switch in Fig. 5 and $P\left(t^{*}\right)$ is used as reference.

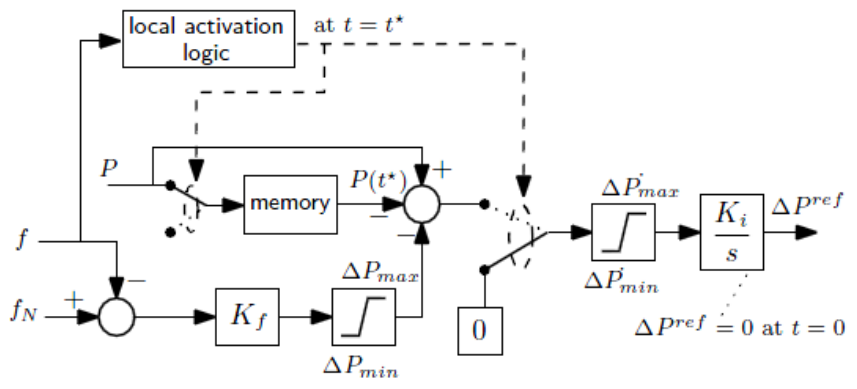

Figure 5: Frequency support control

Assuming that no limitation is active, the integral control forces in steady-state:

$$
P(t \rightarrow \infty)-P\left(t^{*}\right)-K_{f}\left(f_{N}-f(t \rightarrow \infty)\right)=0
$$

where $f_{N}$ denotes the nominal frequency and $K_{f}$ is the gain shown in Fig. 5. Clearly, this relationship is similar to the speed droop characteristic of a conventional speed governor.

The proposed frequency control is intended to act on VSCs in active power control or DC voltage droop control mode (see Fig. 4). When activated, it takes over these controls since the power $P$ settles at a value dictated by Eq. (8).

After a steady-state is reached, the control is deactivated by setting the switch in Fig. 5 back to its original position. Thus, $\Delta P^{r e f}$ will remain constant until a new disturbance occurs in the area the VSC is connected to, or until a centralized control takes action to reset it to zero.

The interaction between the various control modes is illustrated on the simple example of Fig. 6. The asynchronous systems A and B are connected through a HVDC grid with four converters operating in DC voltage-droop mode. VSC1 and VSC2 (resp. VSC3 and VSC4) monitor the frequency of system A (resp. B). If a generator outage in B causes a frequency drop that activates the frequency control on VSC3 and VSC4, both of them inject power in $\mathrm{B}$, emulating the response of two power plants. This injection is backed by VSC1 and VSC2 through the DC voltage droop mechanism. In turn, this power is provided by the power plants of A under frequency control.

Note that if VSC3, for instance, was not provided with the supplementary frequency control, it would participate in the DC voltage control regulation of the HVDC grid (owing to the DC voltage-droop control) and would transfer to the HVDC grid a fraction of the power drawn from it by VSC4. The outcome would be a reduced power support to system B. This counteracting response could be avoided by operating VSC3 in power control but, in this case, this converter would not participate in sharing with VSC4 the power requested by VSC1 and VSC2 after an under-frequency event in area A.

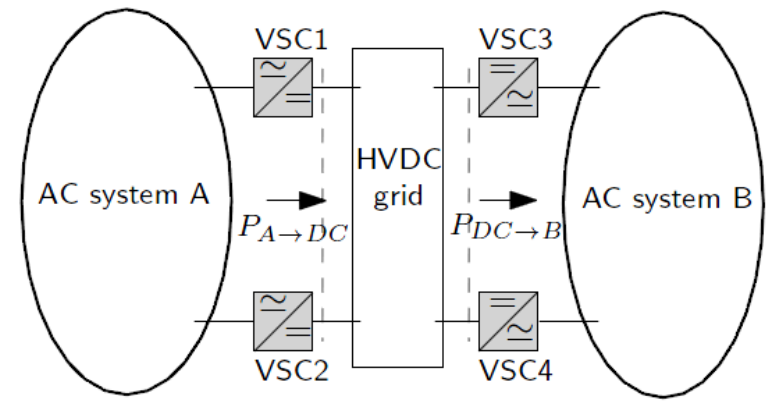

Figure 6: Simple illustrative system

A closer look at the power balancing mechanism is proposed in Fig. 7. If the resistances of all HVDC components are neglected for simplicity, the DC voltages are the same at all nodes. Hence the PV curves of the various VSCs can be combined as shown in Fig. 7, where the straight line with negative (resp. positive) slope refers to the power $P_{A \rightarrow D C}$ transferred from A to DC grid (resp. $P_{D C \rightarrow B}$ transferred from DC grid to $\mathrm{B})$. The initial operating point is $\mathrm{K}$, at the intersection between both characteristics.

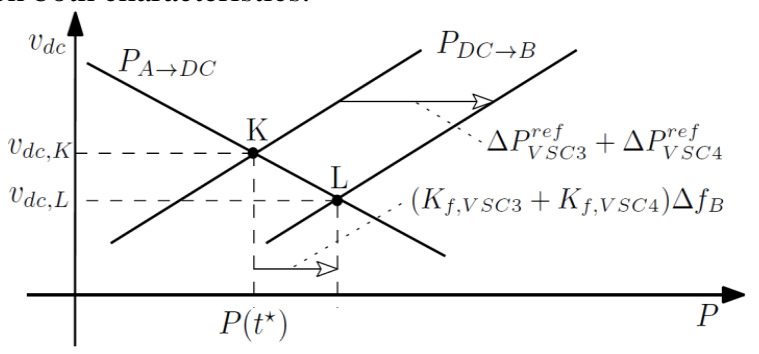

Figure 7: Power balancing mechanism

Following an event in system $B$ resulting in a final frequency drop $\Delta f_{B}$, the power transfer from $\mathrm{A}$ to $\mathrm{B}$ increases by $\left(K_{f, V S C 3}+K_{f, V S C 4}\right) \Delta f_{B}$ leading to operating point L. Under the effect of the integral control, the characteristic $P_{D C \rightarrow B}$ is shifted to the right by the amount $\Delta P_{V S C 3}^{r e f}+\Delta P_{V S C 4}^{r e f}$, as shown in the figure. This amount can be computed as:

$$
\begin{aligned}
\Delta P_{V S C 3}^{r e f}+\Delta P_{V S C 4}^{r e f}= & \left(K_{f, V S C 3}+K_{f, V S C 4}\right) \Delta f_{B} \\
& +\left(K_{v, V S C 3}+K_{v, V S C 4}\right)\left(v_{d c, K}-v_{d c, L}\right)
\end{aligned}
$$

which shows that the DC voltage-droop effect has to be counteracted to achieve the desired participation.

\section{Tuning the controller parameters}

The choice of the various parameters involved in the scheme of Fig. 5 is discussed hereafter.

\subsection{Choice of $\Delta P_{\min }, \Delta P_{\max }, \Delta \dot{P}_{\min }^{\cdot}$ and $\Delta \dot{P}_{\max }^{\cdot}$}

$\Delta P_{\text {max }}$ is the maximum power increase by the VSC in response to a frequency drop. It plays the role of the primary reserve in a conventional power plant. Obviously, the largest possible value is dictated by the thermal limit of the converter. However, a smaller value may be enforced in case the oth- 
er AC systems have a limited support capability, for instance when a "small" system is asked to support the frequency of a "large" one. The (optional) limit $\Delta P_{\max }^{\cdot}$ on the rate of change of power has a similar motivation, i.e. avoid impacting the other AC systems with a fast changing power request, for instance in the case where the power plants have a limited response speed.

$\Delta P_{\text {min }}$ and $\Delta P_{\text {min }}^{\cdot}$ are the corresponding (negative) limits in response to a frequency rise.

\subsection{Choice of $K_{f}$}

With the introduction of integral control, and assuming that the limits $\Delta P_{\text {min }}$ and $\Delta P_{\max }$ (or a DC line thermal limit) are not reached, the steady-state participation of the VSC to frequency regulation is defined by the $K_{f}$ parameter exclusively. Thus, $K_{f}$ can be chosen according to the desired steady-state participation of the VSC to frequency regulation. A small value yields a small participation, and conversely.

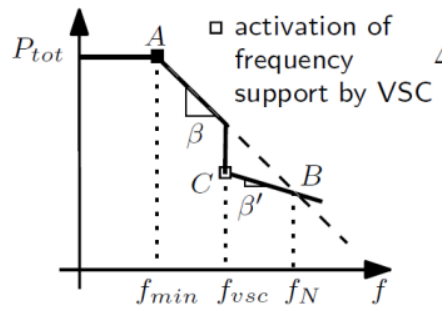

(a) $f$-P characteristic of $\mathrm{AC}$ system

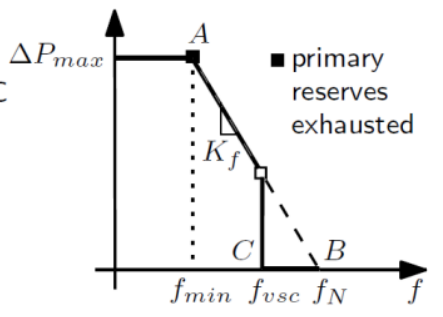

(b) $f$-P characteristic of VSC
Figure $8: f-P$ characteristics for tuning the $K_{f}$ parameter

The selection of $K_{f}$ can rely on the regulating energy (also referred to as "network power frequency characteristic" [11]). The case of a single VSC is considered for simplicity but extension to several frequency supporting VSCs is straightforward. If the VSC would operate without deadband, the overall power vs. frequency characteristic would be the line AB shown in Fig. 8(a), characterized by a regulating energy $\beta$. In this figure, it is assumed for simplicity that all primary reserves (including the one of the VSC) are exhausted at the same frequency $f_{\text {min }}$. In fact, due to its deadband, the VSC only acts for frequencies lower than $f_{v s c}$. For higher values, i.e. for smaller frequency variations, the system is characterized by a lower regulating energy $\beta$, , corresponding to the segment BC in Fig. 8(a). Once the VSC support is activated the steady-state operating point gets back to the $\mathrm{AB}$ characteristic, on which it remains until the VSC frequency control is deactivated. Figure 8(b) shows the corresponding participation of the VSC. From this figure, it is easily derived that:

$$
K_{f}\left(f_{v s c}-f_{\text {min }}\right)=\Delta P_{\text {max }}-K_{f}\left(f_{N}-f_{v s c}\right)
$$

from which the value of $K_{f}$ is easily found as:

$$
K_{f}=\frac{\Delta P_{\max }}{f_{N}-f_{\min }}
$$

Let us repeat that this simple way of choosing $K_{f}$ assumes all primary reserves are exhausted at the same frequency.

\subsection{Choice of $K_{i}$}

Following the selection of the frequency droop $K_{f}$, the integral gain $K_{i}$ is tuned. This degree of freedom is used to achieve the desired dynamic response to frequency transients. Clearly, larger $K_{i}$ values will yield faster power changes, which can help mitigating the frequency dip after a disturbance, and fast responses can be supported by the power electronics of VSCs. However, since the steady-state contribution of the VSC is dictated by $K_{f}$ only, a high initial response will lead to an overshoot of the $P(t)$ evolution, which in turn will impact the other AC systems. Furthermore, as already mentioned, a fast initial change of the VSC power will draw on the energy stored in the DC capacitor, leading to an undesired dip in the DC voltage.

\section{Simulation results}

\subsection{System description}

The aforementioned scheme has been tested on a system consisting of two asynchronous AC areas and an offshore wind farm, connected through an HVDC grid. Each AC system is based on the Nordic test system detailed in [15]. The HVDC grid includes five VSC terminals, of which two are connected to each AC system and one to the wind farm. The one-line diagram of the whole system is shown in Fig. 9, with the two AC systems identified as "Nordic West" and "Nordic East".

With respect to Ref. [15] the following changes were made:

- the large, equivalent generator connected to bus 4072 (becoming $4072 \mathrm{~W}$ and $4072 \mathrm{E}$, respectively, in the mirrored systems) has been removed and replaced by converters VSC2E and VSC2W, respectively (see Fig. 9);

- the power flow data have been adjusted to create a transfer from West to East through the HVDC grid;

in the Eastern system, the thermal power plants of the Central area have been modelled with a three-stage steam turbine and a speed governor. Furthermore, a fraction of their active power production has been transferred to the hydro units of the North region in order to leave them with a reserve for primary frequency regulation. Thus, from the frequency control viewpoint, the Eastern system behaves as a hydro-thermal system, while the West system is entirely controlled by the hydro units of the North W region.

The data of the HVDC cables and VSCs are given in Tables 1 and 2, respectively. All VSCs have identical parameters except for their capacitances which account for the shunt capacitances of HVDC cables (see Fig. 1). The initial HVDC grid operating point is given in Table 3 , where powers are counted positive when drawn from the HVDC grid.

For simplicity, VSC1, VSC2E and VSC2W are assumed to operate in constant power mode, while VSC3E and VSC3W (see the dash-dotted circles in Fig. 9) are in DC voltage-droop mode with $K_{v}=10 \mathrm{MW} / \mathrm{kV}$. The latter two VSCs are also provided with the proposed frequency support control. The wind farm is modelled as a constant power injection. 


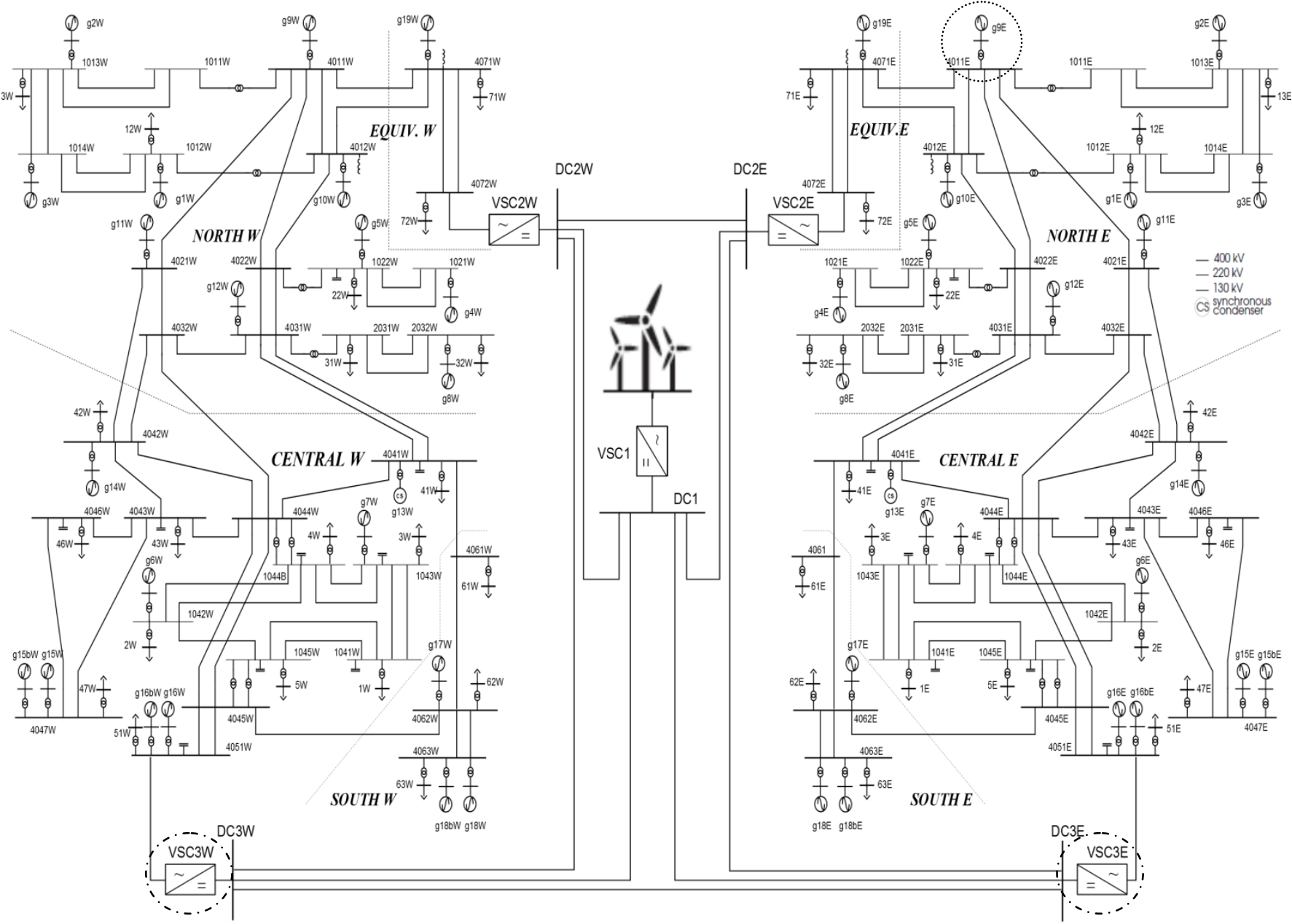

Figure 9: One-line diagram of AC/DC test system

\begin{tabular}{|c|c|c|c|c|}
\hline Name & Bus 1 & Bus 2 & $\mathrm{P}_{\text {nom }}(\mathrm{MW})$ & $\mathrm{R}(\Omega)$ \\
\hline HVDC1 & DC1 & DC2E & 800 & 3.575 \\
HVDC1B & DC1 & DC2W & 800 & 3.575 \\
HVDC2 & DC1 & DC3E & 800 & 2.2 \\
HVDC2B & DC1 & DC3W & 800 & 2.2 \\
HVDC3 & DC2E & DC2W & 800 & 3.025 \\
HVDC4A & DC2E & DC3E & 800 & 8.8 \\
HVDC4B & DC2W & DC3W & 800 & 8.8 \\
HVDC5 & DC3E & DC3W & 800 & 3.3 \\
\hline
\end{tabular}

Table 1: HVDC cable parameters

\begin{tabular}{|c|c|c|c|c|c|}
\hline Parameter & VSC1 & VSC2E & VSC2W & VSC3E & VSC3W \\
\hline $\mathrm{P}_{\text {nom }}(\mathrm{MW})$ & 1000 & 1000 & 1000 & 1000 & 1000 \\
$\mathrm{~V}_{\text {nom }}^{\mathrm{dc}}(\mathrm{kV})$ & 320 & 320 & 320 & 320 & 320 \\
$\mathrm{R}_{\mathrm{r}}(\Omega)$ & 0.48 & 0.48 & 0.48 & 0.48 & 0.48 \\
$\mathrm{~L}_{\mathrm{r}}(\mathrm{mH})$ & 0.045 & 0.045 & 0.045 & 0.045 & 0.045 \\
$\mathrm{C}_{\mathrm{dc}}(\mu \mathrm{F})$ & 250 & 260 & 260 & 240 & 240 \\
\hline
\end{tabular}

Table 2: VSC parameters

As regards the proposed control, the limit $\Delta P_{\max }$ was assumed to be $420 \mathrm{MW}$, and the activation frequency $f_{v s c}$ was set to $0.996 \mathrm{pu}$. At the considered operating point, generator g7E is the last to have its primary reserve exhausted, at $f_{\min }=$ $0.979 \mathrm{pu}$. Thus, Eq. (11) yields $K_{f}=20 \mathrm{pu} / \mathrm{pu}$ (on the con- verter MW base). The tuning of the other parameters is discussed in the next sub-section.

\begin{tabular}{|c|c|c|}
\hline VSC & $\mathrm{P}(\mathrm{MW})$ & $\mathrm{V}_{\mathrm{dc}}(\mathrm{pu})$ \\
\hline VSC1 & -151.8 & 1.00000 \\
VSC2E & -100 & 1.00122 \\
VSC2W & -250 & 1.0021 \\
VSC3E & 400 & 0.9975 \\
VSC3W & 100 & 0.9988 \\
\hline
\end{tabular}

Table 3: HVDC operating point (power flow calculation performed with VSC1 covering the DC losses)

All (phasor-mode) time simulations were performed with the RAMSES software developed at the Univ. of Liège [16].

\subsection{Simulation results}

The results shown hereafter relate to the outage of g9E (identified with the dotted circle in Fig. 9), the largest generator of the Eastern system. The disturbance is applied at $t=10 \mathrm{~s}$. The frequency response of the Eastern system is shown in Fig. 10. The lower solid grey line relates to the case without frequency support from HVDC; the imbalance is covered only by the generators of the East subsystem, leaving the power exchange with the DC grid unchanged. Frequency support by VSC3E is 
activated as soon as frequency drops below the $f_{s v c}=0.996$ pu threshold. This yields a less pronounced frequency dip and a higher final frequency value. Increasing the value of $K_{i}$ does not affect the steady-state frequency, as expected, but reduces the frequency dip, due to the faster reaction of the VSC to the frequency transient.

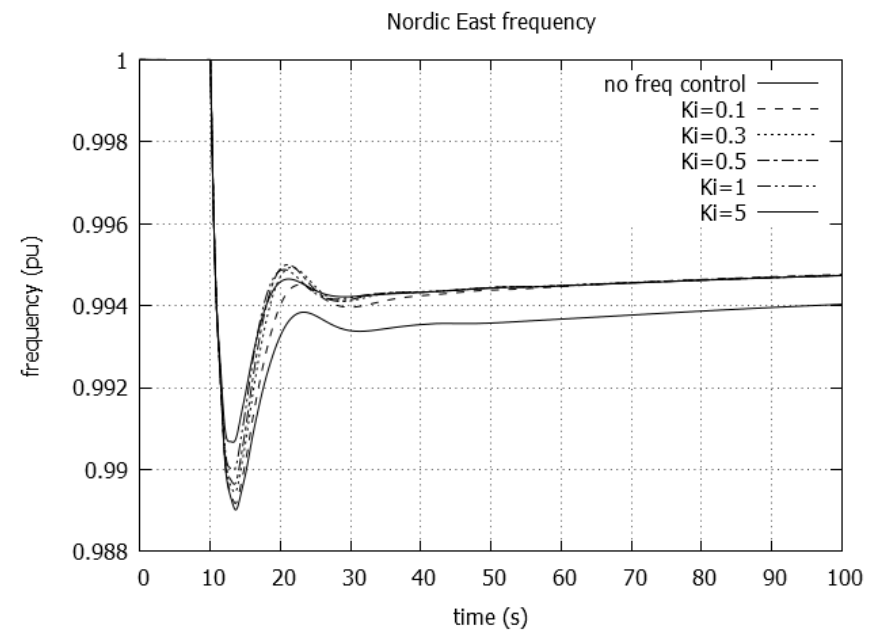

Figure 10: East frequency response for various $K_{i}$ values

As shown in Fig. 11, increasing $K_{i}$ speeds up the variation of the VSC power. For low $K_{i}$ values, the power smoothly reaches the desired steady-state value, while as $K_{i}$ is increased the evolution follows that of frequency more and more closely. However, this results in a larger overshoot taking place when frequency reaches its minimum. The dotted horizontal line in Fig. 11 corresponds to an overshoot of $25 \%$ of the final participation of the VSC. If this is taken as the maximum allowed value, then the optimal value of $K_{i}$ lies in between 0.3 and 0.5 .

It should be noted that the speed of variation of the VSC3E power does not keep on increasing with the integral gain, but saturates beyond some value. The reason is that the proposed integral control cannot be faster than the proportional control it enforces. As a result, even by making the controller almost instantaneous, the speed of response of the proposed control would be upper-bounded by the speed of response of a VSC equipped with a simple frequency droop control scheme and no participation in the HVDC grid active power sharing $\left(K_{v}=0\right)$.

In Fig. 12, the power response of VSC3E is compared with that of the thermal power plant $\mathrm{g} 16 \mathrm{E}$, when $K_{i}$ is set to 0.3 . It can be seen that a similar speed of response has been achieved. In other words, the converter emulates a power plant. Moreover, it has to be noted that the electromechanical oscillations of the AC system are not observed in the VSC3E response, despite it is located next to $\mathrm{g} 16 \mathrm{E}$.

Without communication between the VSCs connected to the HVDC grid, the increase of the VSC3E power causes a power imbalance at HVDC grid level. Consequently, the DC capacitors start discharging and DC voltages decrease, as confirmed by Fig.13, relative to the DC bus DC3E (the response is similar at the other DC buses). The power overshoot experienced on the AC side for large values of $K_{i}$ is reflected on the DC side as an undesired voltage dip.

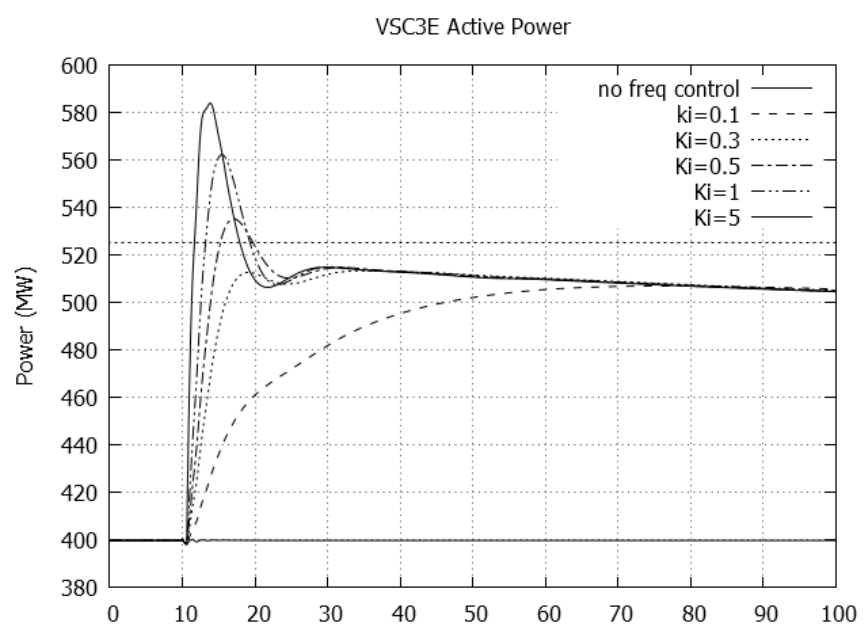

Figure 11: VSC3E power response for various $K_{i}$ values

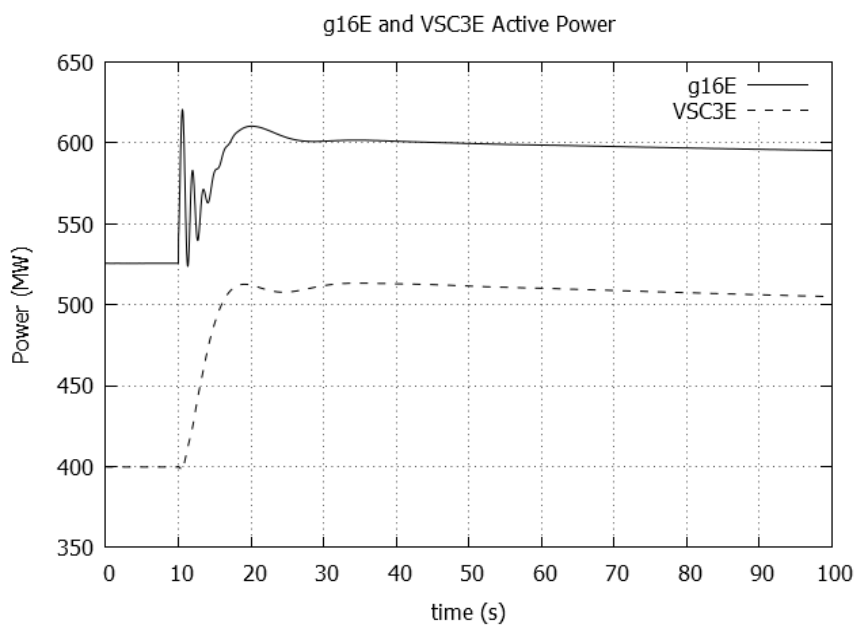

Figure 12: VSC3E and g16E power responses for $K_{i}=0.3$

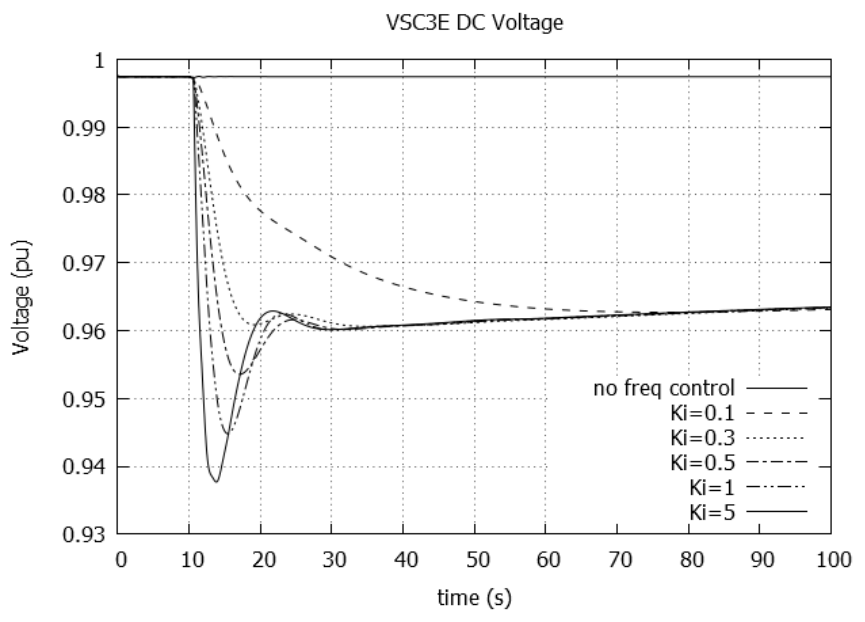

Figure 13: DC bus voltage response for various $K_{i}$ values 


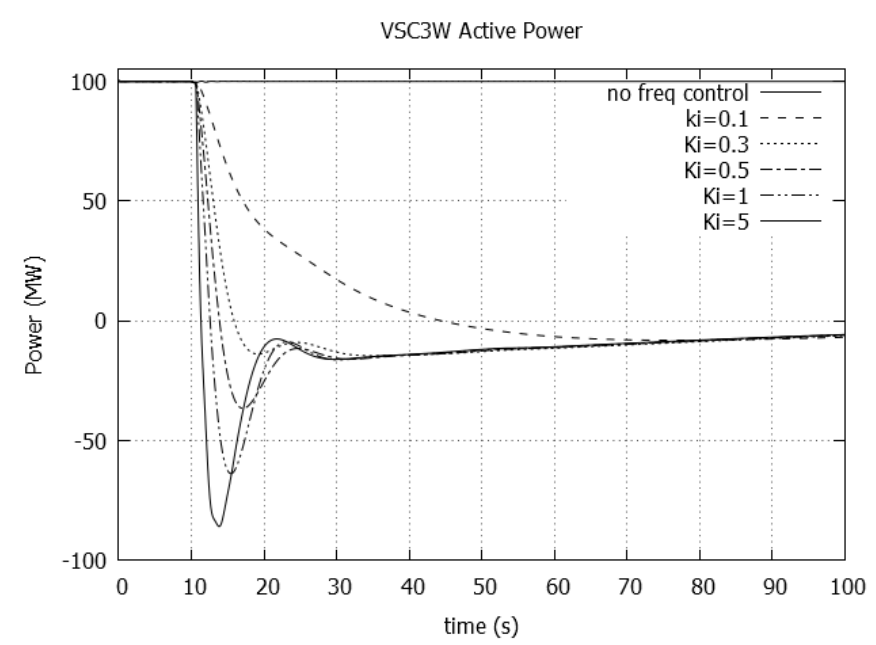

Figure 14: VSC3W power response for various $K_{i}$ values

Then, through the DC voltage-droop control, VSC3W modifies its power until a new equilibrium is reached. This is confirmed by Fig.14. Since the voltage-droop control is almost instantaneous and the capacitance of the DC grid is small, the response of the DC voltage is in the order of a few hundred milliseconds, i.e. it follows almost instantaneously the evolution of the VSC3E power. The same holds true for the response of the VSC3W power. Eventually, VSC3W supplies the whole power requested by VSC3E for frequency support.

As previously mentioned, the introduction of frequency control through the HVDC grid propagates some disturbance into the asynchronously operating, and not initially affected area. This can be observed in Fig. 15 showing the frequency evolution in the West system. The reaction of VSC3W is felt as a load increase in the West system, and is covered by its own conventional power plants. The higher the value of the integral gain $K_{i}$, the faster and the more pronounced the frequency drop in the West system.

To prevent triggering undesired effects in the West system (such as Rate Of Change Of Frequency relays), the rate limit

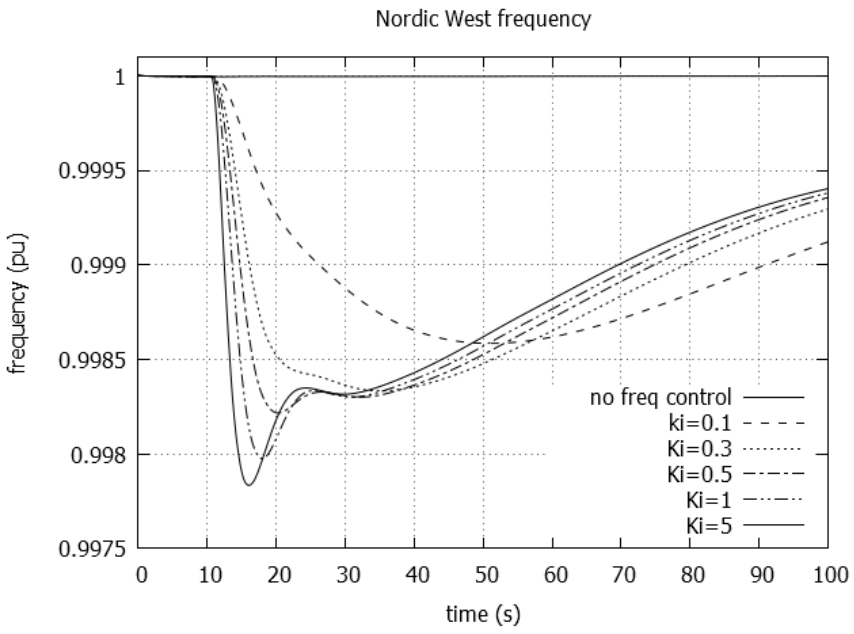

Figure 15: West frequency response for various $K_{i}$ values
$\Delta P_{\text {max }}^{\cdot}$ (see Fig. 5) can be imposed on VSC3E. This ensures that the frequency support provided to the East subsystem will respect the transient constraints set by the West system and vice versa. This way it contributes to mitigating the propagation of the disturbance. As an example, the evolutions of the VSC3E power and the West system frequency are shown in Figs 16 and 17, respectively, both without limit and for $\Delta P_{\max }^{\cdot}$ set to a small $(25 \mathrm{MW} / \mathrm{s})$ value. $K_{i}$ was set to 0.3 in both cases. Note that the effective rate of change of power observed in Fig.16 is smaller than $\Delta P_{\max }^{\cdot}$. The reason is that the DC voltage droop control of VSC3E remains active and continues influencing the active power output of the VSC. The effective rate of change of power depends on the number, the topology and the voltage-droop gains of the VSCs participating in voltage control of the HVDC grid. This observation also implies that the selection of $K_{i}$ can be different in case of a different power sharing strategy of the HVDC grid, since this strategy affects the speed of the VSC frequency response.

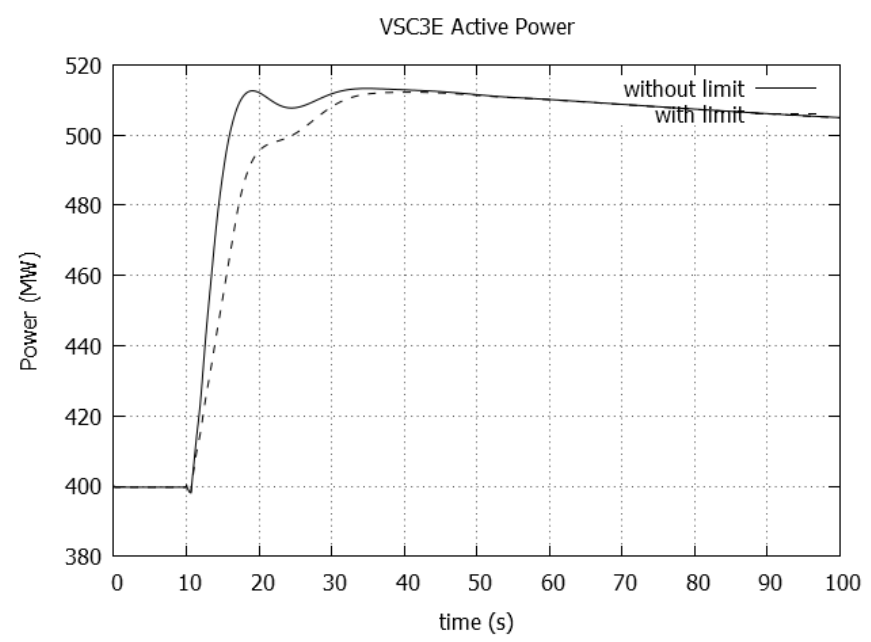

Figure 16: VSC3E power with and without rate limit $\Delta P_{\max }^{\cdot}$

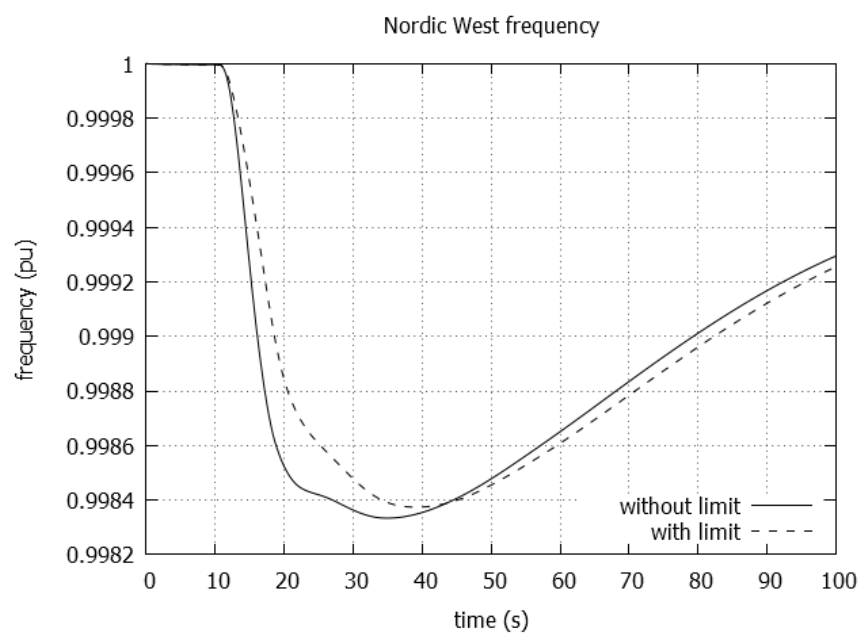

Figure 17: West frequency with and without rate limit $\Delta P_{\max }^{\cdot}$

\section{Conclusion}

A new scheme for mutual frequency support between asynchronous AC systems has been proposed and described. The 
scheme is designed in the spirit of an emergency control, acting for large enough frequency deviations.

In contrast to the simple proportional droop control, this scheme relies on integral control to progressively enforce the desired participation of the VSC to frequency regulation. The steady-state participation of the VSC to frequency regulation can be chosen using the well-known concept of regulating energy. The desired transient response can be achieved by properly adjusting the integral gain. The method illustrated in this paper consists of slowing down the VSC response to limit not only the power overshoot but also the DC voltage dip (and, hence, the stress applied to the HVDC system).

Simulation results on a two-area system confirmed the effectiveness of the proposed control. The mechanism of power transfer from one system to the other was highlighted, as well as the interaction between the two AC systems, and a measure to limit the propagation of the initial disturbance was considered.

Future research will further investigate the interactions between the two areas and will focus on the HVDC system behaviour when more or all the VSCs participate to frequency support, in addition to DC voltage-droop control. Moreover, the overall impact of the HVDC grid on the robustness of the AC systems will be examined.

\section{Acknowledgements}

The authors thank Patrick Panciatici and Marie-Sophie Debry of RTE, France, for their valuable comments and suggestions.

\section{References}

[1] "Ten year network development plant 2010-2020", technical report, ENTSO-E, 2010.

[2] D. Van Hertem, M. Ghandhari. "Multi-terminal VSC HVDC for the European supergrid: Obstacles," Renewable and Sustainable Energy Reviews, 14, pp.31563163, (2010).

[3] L. Bizumic, R. Cherkaoui, U. Häger. "Interface Protection," in Monitoring, Control and Protection of Interconnected Power Systems, Springer, pp. 333-347, (2014).

[4] J. Dai, Y. Phulpin, A. Sarlette, D. Ernst. "Voltage control in an HVDC system to share primary frequency reserves between non-synchronous areas," in Proc. Power System Computation Conference (PSCC), (2011).

[5] T. M. Haileselassie, K. Uhlen. "Primary Frequency Control of Remote Grids Connected by Multi-terminal HVDC," in Proc. IEEE PES General Meeting, (2010).

[6] N. R. Chaudhuri, R. Majumder, B. Chaudhuri. "System Frequency Support Through Multi-Terminal (MTDC) Grids," IEEE Transactions on Power Systems, 28, pp. 347-356, (2013).

[7] Y. Phulpin. "Communication-free Inertia and Frequency Control for Wind Generators Connected by an HVDC-
Link," IEEE Transactions on Power Systems, 27, pp.1136-1137,(2012).

[8] B. Silva, C. L. Moreira, L. Seca, J. A. Peças Lopes. "Provision of Inertial and Primary Frequency Control Services Using Offshore Multiterminal HVDC Networks," IEEE Transactions on Sustainable Energy, 3, (2012).

[9] T. K. Vrana, L. Zeni, O. B. Fosso. "Active Power Control with Undead-Band Voltage \& Frequency Droop Applied to a Meshed DC Grid Test System," in Proc. IEEE International ENERGYCON, (2012).

[10] C. E. Spallarossa, Y. Pipelzadeh, T. C. Green. "Influence of Frequency-Droop Supplementary Control on Disturbance Propagation through VSC HVDC Links," in Proc. IEEE PES General Meeting, (2013).

[11] P. Kundur. Power System Stability and Control, McGraw-Hill, 1994.

[12] S. Cole, J. Beerten, R. Belmans. "Generalized Dynamic VSC MTDC Model for Power System Stability Studies," IEEE Transactions on Power Systems, 25, pp. 1655-1662, (2010).

[13] P. Rault, X. Guillaud, F. Colas, S. Nguefeu. "Investigation on interactions between AC and DC grids," in Proc. IEEE Powertech conference, (2013).

[14] P. Rault, F. Colas, X. Guillaud, S. Nguefeu. "Method for Small Signal Stability Analysis of VSC-MTDC grids," in Proc. IEEE PES General Meeting, (2012).

[15] T. Van Cutsem, L. N. Papangelis. "Description, Modeling and Simulation Results of a Test System for Voltage Stability Analysis," internal report, Univ. of Liège, (2013). Available at http://hdl.handle.net/2268/141234

[16] P. Aristidou, D. Fabozzi, and T. Van Cutsem. "Dynamic simulation of large-scale power systems using a parallel Schur-complement-based decomposition method," IEEE Transactions on Parallel and Distributed Systems, (2013). Available at http://hdl.handle.net/2268/156230 